\title{
Uptake and tumor-suppressive pathways of exosome-associated GKN1 protein in gastric epithelial cells
}

\author{
Jung Hwan Yoon ${ }^{1} \cdot$ Hassan Ashktorab ${ }^{2}$. Duane T. Smoot ${ }^{3} \cdot$ Suk Woo Nam $^{1} \cdot$ Hoon Hur ${ }^{4} \cdot$ Won Sang Park ${ }^{1}$
}

Received: 29 January 2020 / Accepted: 26 March 2020 / Published online: 6 April 2020

(c) The International Gastric Cancer Association and The Japanese Gastric Cancer Association 2020

\begin{abstract}
Background Gastrokine 1 (GKN1) is a stomach-specific tumor suppressor that is secreted into extracellular space as an exosomal cargo protein. The objective of this study was to investigate the uptake and tumor-suppressive pathways of exosomeassociated GKN1 protein in gastric epithelial cells.

Methods Immunofluorescent and Western blot analysis were used to investigate gastric-specific uptake of HFE-145-derived exosomes. Binding affinity of HFE-145 derived exosomes with integrin proteins was examined using protein microarray chip. Tumor suppressor activities of exosome-carrying GKN1 protein were analyzed using transwell co-culture, MTT assay, BrdU incorporation, immunoprecipitation, and Western blot analysis.

Results HFE-145-derived exosomes were internalized only into HFE-145 gastric epithelial cells and gastric cancer cells. Gastric-specific uptake of stomach-derived exosomes required integrin $\alpha 6$ and $\alpha \mathrm{X}$ proteins. Clathrin and macropinocytosis increased the uptake of exosomes into gastric epithelial cells, whereas caveolin inhibited the uptake of exosomes. Transwell co-culture of AGS cells with HFE-145 cells markedly inhibited viability and proliferation of AGS cells. Following uptake of HFE-145-derived exosomes in recipient cells, GKN1 protein bound to HRas and inhibited the binding of HRas to b-Raf and c-Raf which subsequently downregulated HRas/Raf/MEK/ERK signaling pathways in AGS, MKN1 cells, and MKN1derived xenograft tumor tissues. In addition, exosomal GKN1 protein suppressed both migration and invasion of gastric cancer cells by inhibiting epithelial-mesenchymal transition.

Conclusions Gastric-specific uptake of exosomes derived from gastric epithelial cells requires integrin $\alpha 6$ and $\alpha \mathrm{X}$ proteins in both gastric epithelial cells and exosomes. Exosomal GKN1 protein inhibits gastric carcinogenesis by downregulating HRas/Raf/MEK/ERK signaling pathways.
\end{abstract}

Keywords GKN1 $\cdot$ Exosome $\cdot$ Uptake $\cdot$ Ras signaling $\cdot$ Gastric cancer

Electronic supplementary material The online version of this article (https://doi.org/10.1007/s10120-020-01068-2) contains supplementary material, which is available to authorized users.

Won Sang Park

wonsang@catholic.ac.kr

1 Department of Pathology, Functional RNomics Research Center, College of Medicine, The Catholic University of Korea, 222 Banpo-daero, Seocho-gu, Seoul 06591, South Korea

2 Department of Medicine, Howard University, District of Columbia, Washington 20060, USA

3 Department of Medicine, Meharry Medical Center, Nashville, TN 37208, USA

4 Department of Surgery, Brain Korea 21 Plus Research Center for Biomedical Science, Ajou University School of Medicine, Suwon 16499, South Korea

\section{Introduction}

Exosomes are membrane-bound extracellular vesicles of $40-100 \mathrm{~nm}$ in diameter. They are secreted from all most cell types into the extracellular environment $[1,2]$. In general, exosomes enclose a variety of contents, such as DNA, mRNA, miRNA, proteins, and bioactive lipids $[3,4]$, suggesting that exosomes play an important role in intercellular communication. Because exosomal cargo is a potential communicative agent, normal cell-derived exosomes are known to block key signaling pathways involved in cancer by tumor-suppressive contents, whereas oncogenic contents of tumor exosomes cause malignant transformation of recipient cells [5, 6]. Notably, proteins on the surface of exosomes have been reported to affect the uptake rate of exosomes into recipient cells [7]. Due to high organotropism of exosomes, exosome-based drug 
delivery systems could resolve insufficient targeting efficiency and translation difficulty. Previously, Hoshino et al. [8] have reported that integrins in exosomes can induce organ-specific colonization in a tissue-specific fashion. Integrins play a role in exosome adhesion to target cells by forming heterobimolecular complexes with tetraspanins such as CD9, CD63, and CD81 $[9,10]$. Recent study has demonstrated that integrin $\alpha_{v}$ and $\beta_{4}$ are increased in exosomes from cancer patients with liver metastasis and lung metastasis, respectively, suggesting that integrin expression pattern is closely correlated with organotropism of exosome uptake [8]. In addition, the biological activity of exosomes that can interact with and be absorbed by specific cells is adjusted by various types of endocytosis such as phagocytosis [11], macropinocytosis [12], clathrin-mediated [13], caveolin-mediated [14], clathrin/caveolin-independent [15], and lipid raft-mediated [16] endocytosis. Although many studies have revealed the role of exosomes in cancer development and progression, the cell type-specific uptake pathways of exosomes are still unclear. Elucidating the mechanism underlying exosome internalization is important for understanding organ-specific targeting process of exosomes.

Human gastrokine 1 (GKN1), a stomach-specific protein, is produced by gastric mucosal epithelium, stored in cytoplasmic granules, and released into the extracellular environment as an exosomal cargo protein [17-19]. Notably, the role of GKN1 consists of maintaining mucosal integrity and regulating cell differentiation [20, 21]. We have previously demonstrated frequent absence of GKN1 expression in gastric cancer cells and tumor-suppressive activities of GKN1 by inhibiting cell proliferation, epithelial-mesenchymal transition (EMT), and cell migration [21-24]. In particular, GKN1-positive exosomes isolated from HFE-145 immortalized gastric epithelial cells can dramatically suppress cell growth and lead to cell cycle arrest and apoptotic cell death of AGS and MKN1 gastric cancer cells in vitro and in vivo studies [18]. Because exosomal secretion of proteins is expected to alter the phenotype and function of recipient cells, it is very important to understand roles of exosomal proteins in normal physiological processes and tumorigenesis.

Here, we focused on how exosomes derived from normal gastric epithelial cells and gastric cancer cells were specifically internalized into gastric cells and molecular pathways underlying tumor suppressor activity of exosomal GKN1 protein.

\section{Materials and methods}

\section{Cell culture and transfection of integrins}

AGS gastric cancer cells, MKN1 gastric cancer cells, HT29 colon cancer cells, H460 lung cancer cells, and SNU449 hepatocellular carcinoma cells were grown in RPMI-1640 medium (Lonza, Basel, Switzerland) supplemented with $10 \%$ heat-inactivated fetal bovine serum at $37{ }^{\circ} \mathrm{C}$ with $5 \%$ $\mathrm{CO}_{2}$. These cells did not express GKN1 protein. HFE-145 immortalized gastric epithelial cells with expression of GKN1 protein were also cultured in RPMI-1640 medium (Lonza, Basel, Switzerland) supplemented with $10 \%$ heatinactivated FBS at $37{ }^{\circ} \mathrm{C}$ with $5 \% \mathrm{CO}_{2}$. Complete integrin $\alpha 6-, \alpha \mathrm{X}$ - and caveolin 1-cDNA were cloned into the expression vector pcDNA3.1 expression vector (Invitrogen, Carlsbad, CA, USA). AGS, HT29, H460 and SNU449 cells were transiently transfected in $60 \mathrm{~mm}$-diameter dishes with expression plasmids ( $5 \mu \mathrm{g}$ total DNA), using Lipofectamine Plus transfection reagent (Invitrogen) according to the manufacturer's recommendations. In addition, AGS and MKN1 cells were transfected with siIntegrin $\alpha 6$, siIntegrin $\alpha \mathrm{X}$, siClathrin and siCaveolin 1. To clarify the binding domain of GKN1 protein, we produced four deletion-formed plasmids, pGKN1 ${ }^{\Delta 68-199}$ containing the NH2terminal hydrophophic region, $\mathrm{pGKN} 1^{\Delta 1-67,165-199}$ with the BRICHOS domain, pGKN $1^{\Delta 1-164}$ with the $\mathrm{COOH}$-terminus and pGKN $^{\Delta 1-67}$ with the BRICHOS and $\mathrm{COOH}$-terminus [25]. We also generated stably GKN1 knockdown HFE145 cells $\left(\mathrm{HFE}-145^{\mathrm{shKN1}}\right)$ as well as non-targeting shRNA transfectant, HFE- $145^{\text {shCtrl }}$ cells, as described previously [26]. Pitstop 2, genistein, and amiloride were purchased from Sigma-Aldrich (St. Louis, MO, USA).

\section{Co-culture of HFE-145 and AGS cells in transwell systems}

To determine the effect of exosomes derived from HFE-145 gastric epithelial cells on viability and EMT of AGS gastric cancer cells, we developed a transwell cell-culture system as follows. First, we plated $3 \times 10^{3}$ AGS cells onto the bottom of a six-well transwell cell-culture system (Pore size $0.4 \mu \mathrm{m}$; Costar Corp., USA) with complete media and culture environment as described above. We seeded $3 \times 10^{3}$ HFE- 145 cells onto the membrane of the transwell cell-culture inserts and allowed them to grow overnight under the above-mentioned condition. The next day, we washed these cells with serum-free media and cultured them for $24 \mathrm{~h}$ in serum-free medium without growth factors. For co-culture, we moved a membrane transwell insert containing HFE-145 cells into the six-well transwell cell-culture system containing $1 \times 10^{4}$ AGS cells and cultured them for $24 \mathrm{~h}$ in serum-free media. We performed cell viability, migration and invasion experiments after 10 days of culture using the complete media.

\section{Human samples}

A total of five patients with sporadic gastric cancer who underwent a gastrectomy were included. Gastric cancer 
and corresponding non-cancerous gastric mucosae remote $(\geq 5 \mathrm{~cm})$ from the tumor were used in this study. In addition, normal liver $(n=5)$, lung $(n=5)$ and colon $(n=5)$ tissues were enrolled. The Institutional Review Board of The Catholic University of Korea, College of Medicine approved this study (MC16SISI0132).

\section{Exosome isolation}

We isolated exosomes from supernatants from HFE-145 immortalized gastric epithelial cells as described previously [18]. Briefly, cells at passages 3-8 were incubated in serumfree culture medium and supplemented them with $10 \%$ FBS and $1 \%$ penicillin/streptomycin at $37{ }^{\circ} \mathrm{C}$ in a humidified atmosphere with $5 \% \mathrm{CO}_{2}$ for $48 \mathrm{~h}$ before harvesting the medium. To remove cell debris, the medium was centrifuged at $2000 \mathrm{~g}$ for $10 \mathrm{~min}$ at $4{ }^{\circ} \mathrm{C}$ and then passed through a $0.22 \mu \mathrm{m}$ filter. The cleared supernatant was transferred to a new glass tube and placed on ice. The supernatant was mixed with $\mathrm{A} / \mathrm{B} / \mathrm{C}$ solution (101Bio company, CA, USA; $2 \mathrm{ml}$ supernatant with $0.75 \mathrm{ml} \mathrm{A} / \mathrm{B} / \mathrm{C}$ solution) in a new tube, vortexed it for $30 \mathrm{~s}$, and incubated it at $4{ }^{\circ} \mathrm{C}$ for $30 \mathrm{~min}$. The mixture separated into two layers. After removing the top layer, the bottom layer was transferred to a microcentrifuge tube and spun it at $5000 \mathrm{~g}$ for $3 \mathrm{~min}$. The middle fluff layer was transferred to a new tube and we spun it at $5000 \mathrm{~g}$ for $3 \mathrm{~min}$. The cap was left open to air dry for $10 \mathrm{~min}$ at room temperature. We added $4 \times$ volumes of $1 \times \mathrm{PBS}$ to the tube, vigorously pipetted it, placed the tube on a horizontal shaker at high speed for $15 \mathrm{~min}$, and then spun it at $5000 \mathrm{~g}$ for $5 \mathrm{~min}$. We carefully transferred the supernatant to a PureExo® Column (101Bio company, CA, USA) and spun it at $1000 \mathrm{~g}$ for $5 \mathrm{~min}$. The flow-through fraction containing the isolated pure exosome suspended in PBS.

\section{Exosome labeling}

We labeled exosomes with PKH26 (Sigma) as described previously [18]. Briefly, we resuspended exosome pellets in $1 \mathrm{~mL}$ of Diluent C. $1 \mathrm{~mL}$ of Diluent $\mathrm{C}$ was mixed with $4 \mu \mathrm{L}$ of PKH26 and we then mixed the exosome suspension with the stain solution followed by incubation for $20 \mathrm{~min}$ at $37{ }^{\circ} \mathrm{C}$. We stopped the labeling reaction by adding an equal volume of $1 \%$ bovine serum albumin. We isolated the labeled exosomes using a Total Exosome Isolation kit (Invitrogen) according to the manufacturer's protocol. Briefly, we added 0.5 volumes of the isolation reagent to labeled exosomes and vortexed the mixture to mix well. We incubated these labeled exosomes at $4{ }^{\circ} \mathrm{C}$ overnight and subsequently centrifuged them at $10,000 \mathrm{~g}$ for $1 \mathrm{~h}$ at $4{ }^{\circ} \mathrm{C}$. We discarded the supernatant and resuspended each pellet in $100 \mu \mathrm{L}$ of PBS.

\section{Active Ras detection assay}

We seeded gastric cancer cells (AGS and MKN1) into sixwell plates at density of $3 \times 10^{5}$ per well in RPMI-1640 medium. After $24 \mathrm{~h}$, cells were treated with exosomes carrying GKN1 for $48 \mathrm{~h}$. We harvested cells under non-denaturing conditions and rinsed them with cold PBS. Cells were lysed in lysis buffer. An active Ras detection assay kit (Cell Signaling Technology) was used to perform affinity precipitation of active Ras, according to the manufacturer's protocol. We treated cell lysates $(500 \mu \mathrm{g})$ with GTP $\gamma \mathrm{S}$ for activation of Ras, acting as a positive or negative control, respectively. Subsequently, GST-Raf1-RBD fusion protein and cell lysate in glutathione resin were incubated. Electrophoresed and immunoblotting were performed with Ras mouse monoclonal antibody.

\section{Statistical analysis}

Student's $t$ test was used to analyze effects of GKN1 on cell viability and proliferation. We carried out all experiments in duplicate to verify the reproducibility of findings. The results are expressed as means \pm S.D. from two independent experiments. When $P$ value was less than 0.05 , the results were considered statistically significant.

Other methods are described in detail in the Supplementary materials and methods section.

\section{Results}

\section{Cellular uptake of exosomes derived from gastric epithelial cells depends on the origin of the cells}

To investigate whether exosomes derived from gastric epithelial cells were internalized into gastric and non-gastric cells, we isolated exosomes from cultured supernatants from HFE-145 immortalized gastric epithelial cells, AGS and MKN1 gastric cancer cells, H460 non-small-cell lung cancer cells, SNU449 hepatocellular carcinoma cells, and HT29 colorectal adenocarcinoma cells. Intact exosomes with the presence of vesicles ranging in size from 30 to $100 \mathrm{~nm}$ were seen in TEM analysis (Fig. 1a) and the expression of exosomal markers such as CD9, 63, and 81 was observed in exosomes (Fig. 1b). Interestingly, the expression of GKN1 was detected only in exosomes derived from HFE145 immortalized gastric epithelial cells (Fig. 1b). When we treated HFE-145 immortalized gastric epithelial cells, AGS and MKN1 gastric cancer cells, H460 non-small-cell lung cancer cells, SNU449 hepatocellular carcinoma cells, and HT29 colorectal adenocarcinoma cells with exosomes derived from HFE-145 cells, exosomes were seen only in cytoplasms of HFE-145, AGS, and MKN1 cells (Fig. 1c). In 

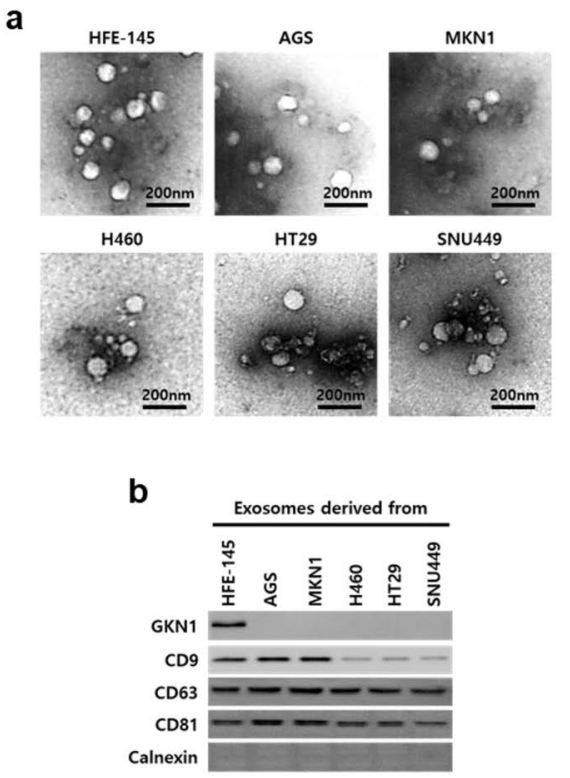
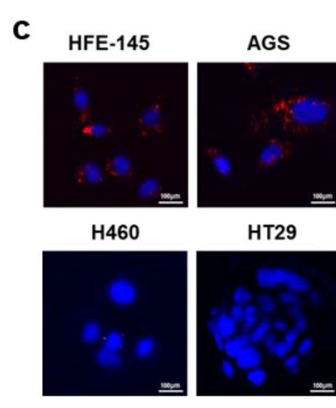

HT29
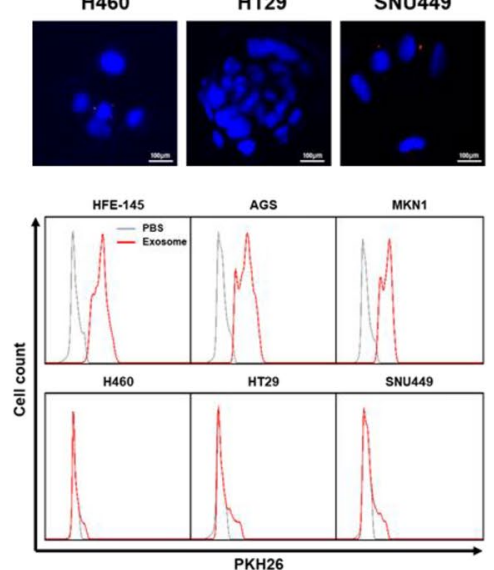

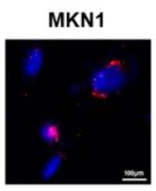

SNU449

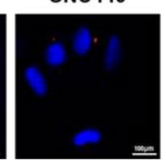

KN1
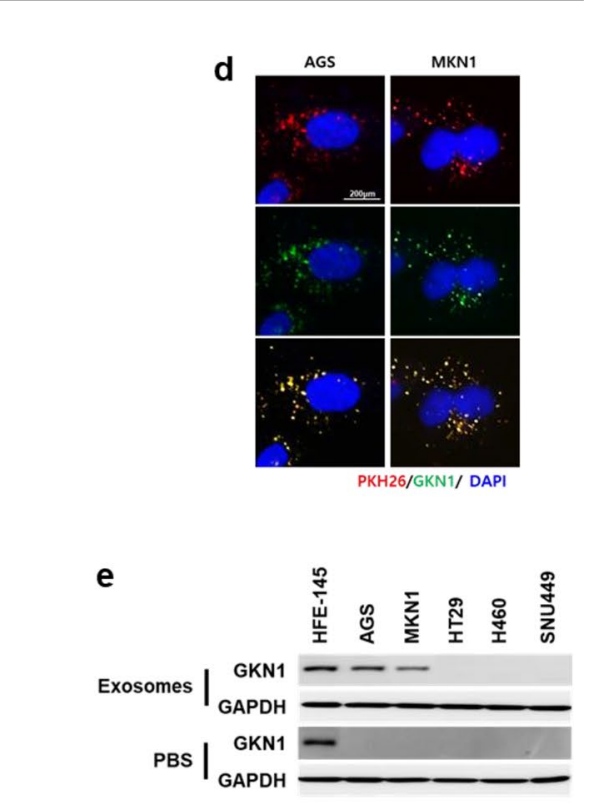

Fig. 1 Cellular uptake of exosomes derived from gastric epithelial cells depends on the origin of the cells. a TEM image of exosomes isolated from supernatants of HFE-145, AGS, MKN1, H460, HT29, and SNU449 cells. b In Western blot analysis, the expression of exosomal markers such as CD9, 63, and 81 was observed in exosomes derived from HFE-145, AGS, MKN1, H460, HT29, and SNU449 cells. However, the expression of GKN1 was only detected in HFE145 cells. c In immunofluorescent and FACS analysis of HFE-145, AGS, MKN1, HT29, H460, and SNU449 cells treated with exosomes

immunofluorescence study, co-localization of GKN1 protein with PKH26 labeled exosomes derived from HFE-145 cells was found in cytoplasms of AGS and MKN1 cells treated with PKH26 labeled exosomes (Fig. 1d). Western blot analysis showed that GKN1 proteins were expressed only in HFE-145, AGS, and MKN1 cells treated with exosomes derived from HFE-145 cells (Fig. 1e), suggesting that HFE145-derived exosomes were only internalized into HFE-145, AGS, and MKN1 cells (Fig. 1e).

\section{Specific uptake of exosomes derived from gastric epithelial cells into the cytoplasm of gastric cells depends on integrin $a 6$ and $a X$}

To elucidate whether gastric-specific uptake of exosomes derived from gastric epithelial cells was associated with integrin proteins, we analyzed the binding affinity of exosomes derived from HFE-145 cells with integrin proteins including integrin $\beta 1, \alpha 6$ and $\alpha \mathrm{X}$ in a protein microarray chip. As shown in Fig. 2a, exosomes had high binding affinity with integrin $\alpha 5, \alpha 11, \alpha \mathrm{X}, \beta \mathrm{L} 1$, and $\beta 1$ (Table 1 ). In addition, we examined integrin protein compositions in HFE145 immortalized gastric epithelial cells, AGS and MKN1 gastric cancer cells, HT29 colorectal adenocarcinoma cells, derived from HFE-145 cells, exosomes were only detected in cytoplasms of HFE-145, AGS, and MKN1 cells. d Co-localization of PKH26 labeled exosomes with GKN1 protein in cytoplasms of AGS and MKN1 gastric cancer cells. e In HFE-145, AGS, MKN1, HT29, H460, and SNU449 cells treated with exosomes derived from HFE145 cells, expression of exosomal GKN1 proteins was observed in HFE-145, AGS, and MKN1, but not in HT29, H460, and SNU449 cells

H460 non-small-cell lung cancer cells, SNU449 hepatocellular carcinoma cells, and exosomes derived from these cells. In HFE-145, AGS, and MKN1 cells, expression levels of integrin $\alpha 5, \alpha 6, \alpha 10, \alpha \varepsilon$, and $\alpha \mathrm{X}$ were higher than those in cancer cells of other tissue origins, although there was no significant difference in integrin protein composition among HFE-145, AGS, and MKN1 cells (Fig. 2b). In particular, integrin $\alpha 6$ and $\alpha \mathrm{X}$ were expressed only in HFE-145, AGS, and MKN1 cells (Fig. 2b). To further confirm these results, we examined the expression levels of integrin $\alpha 6$ and $\alpha \mathrm{X}$ in normal stomach, liver, lung and colon tissues. Consistently, the expression of integrin $\alpha 6$ and $\alpha \mathrm{X}$ proteins was observed only in normal stomach tissues, but not in normal liver, lung and colon tissues (Fig. 2c). There was no significant difference in expression level of integrin $\alpha 6$ and $\alpha \mathrm{X}$ proteins between non-cancerous gastric mucosae and gastric cancer tissues (Fig. 2d). Thus, these results indicate that integrin $\alpha 1, \alpha 4, \alpha \varepsilon, \beta 1$ and $\beta 6$ are required for membrane binding of exosomes derived from gastric epithelial cells. In addition, gastric-specific uptake of exosomes derived from gastric epithelial cells suggests that they are associated with different expression levels of integrin proteins such as integrin $\alpha 6$ and $\alpha \mathrm{X}$ in both gastric cells and exosomes. 

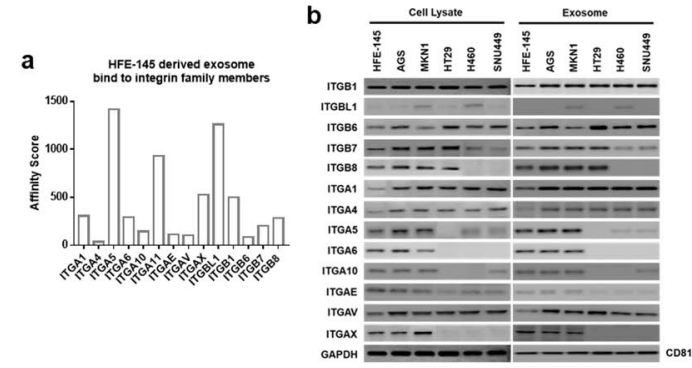

C

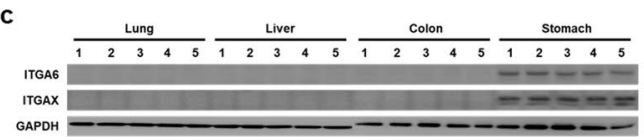

d

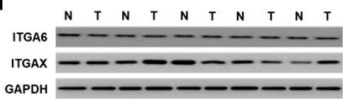

e

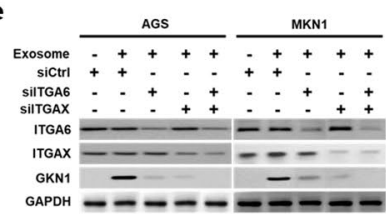

Fig. 2 Specific cellular uptake of exosomes derived from gastric epithelial cells through integrin $\alpha 6$ and $\alpha \mathrm{X}$. a Binding affinity of exosomes derived from HFE-145 cells to integrin proteins including integrin $\alpha 1, \beta 1, \alpha 6$, and $\alpha \mathrm{X}$ in a protein microarray chip. b Western blot analysis showing expression levels of integrin proteins, including integrin $\beta 1, \alpha 6$, and $\alpha \mathrm{X}$, in cell lysates and exosomes from HFE-145, AGS, MKN1, HT29, H460, and SNU449 cells. c The expression of integrin $\alpha 6$ and $\alpha \mathrm{X}$ proteins in normal liver, lung, colon and stomach tissues. d There was no significant difference in the expression of integrin $\alpha 6$ and $\alpha \mathrm{X}$ proteins between corresponding non-cancerous gastric mucosae $(\mathrm{N})$ and gastric cancer $(\mathrm{T})$ tissues. e Knockdown of integrin $\alpha 6$ and $\alpha \mathrm{X}$ in AGS and MKN1 cells treated with exosomes derived from HFE-145 cells reduced expression levels of integrin $\alpha 6$

Next, we examined the effect of integrin $\alpha 6$ and $\alpha \mathrm{X}$ on uptake of exosomes derived from HFE-145 gastric epithelial cells in AGS and MKN1 cells. Knockdown of integrin $\alpha 6$ and $\alpha \mathrm{X}$ with siIntegrin $\alpha 6$ and siIntegrin $\alpha X$ in AGS and MKN1 cells markedly reduced expression levels of integrin $\alpha 6$ and $\alpha \mathrm{X}$, respectively (Fig. 2e). When AGS and MKN1 cells were treated with PKH26labeled exosomes, these exosomes were clearly localized in the cytoplasm of these cells. However, knockdown of integrin $\alpha 6$ and $\alpha \mathrm{X}$ markedly inhibited the internalization of PKH26-labeled exosomes into AGS and MKN1 cells' cytoplasms (Fig. 2f). In addition, level of GKN1 expression was dramatically reduced in AGS and MKN1 cells (Fig. 2e). We further confirmed the role of integrin $\alpha 6$ and $\alpha \mathrm{X}$ in uptake of exosomes derived from gastric epithelial cells in non-gastric cells. In the transient transfection assay performed in HT29 colorectal adenocarcinoma cells, H460 and $\alpha \mathrm{X}$, and GKN1 proteins. $\mathbf{f}$ In immunofluorescent and FACS analysis, knockdown of integrin $\alpha 6$ and $\alpha \mathrm{X}$ inhibited the internalization of exosomes into AGS and MKN1 cells' cytoplasms. g In immunofluorescent and FACS analysis, ectopic expression of integrin $\alpha 6$ and $\alpha \mathrm{X}$ in SNU449, H460, and HT29 cells induced the internalization of exosomes derived from HFE-145 cells into these cells' cytoplasms. h Ectopic expression of integrin $\alpha 6$ and $\alpha \mathrm{X}$ in SNU449, H460, and HT2 29 cells induced expression levels of integrin $\alpha 6$ and $\alpha \mathrm{X}$. In addition, GKN1 protein was detected in these cells treated with exosomes derived from HFE-145 cells. i In immunofluorescent and FACS analysis, exosomes derived from SNU449, H460 and HT29 cells expressing integrin $\alpha 6$ and $\alpha \mathrm{X}$ were detected in the cytoplasm of AGS cells

non-small-cell lung cancer cells, and SNU449 hepatocellular carcinoma cells, ectopic expression of integrin $\alpha 6$ or $\alpha \mathrm{X}$ slightly induced internalization of exosomes derived from HFE-145 gastric epithelial cells (Fig. 2g). However, expression of both integrin $\alpha 6$ and $\alpha \mathrm{X}$ markedly induced internalization of exosomes derived from HFE-145 gastric epithelial cells (Fig. 2g). In Western blot analysis, ectopic expression of both integrin $\alpha 6$ and $\alpha \mathrm{X}$ dramatically increased expression level of GKN1 in SNU449, H460, and HT29 cells (Fig. 2h). Additionally, ectopic expression of integrin $\alpha 6$ and $\alpha \mathrm{X}$ in SNU449, H460, and HT29 cells markedly increased internalization of exosomes derived from these cells into the AGS cells' cytoplasm (Fig. 2i). Thus, these results suggest that integrin $\alpha 6$ and $\alpha \mathrm{X}$ are required for organotropism of exosomes derived from gastric epithelial cells. 


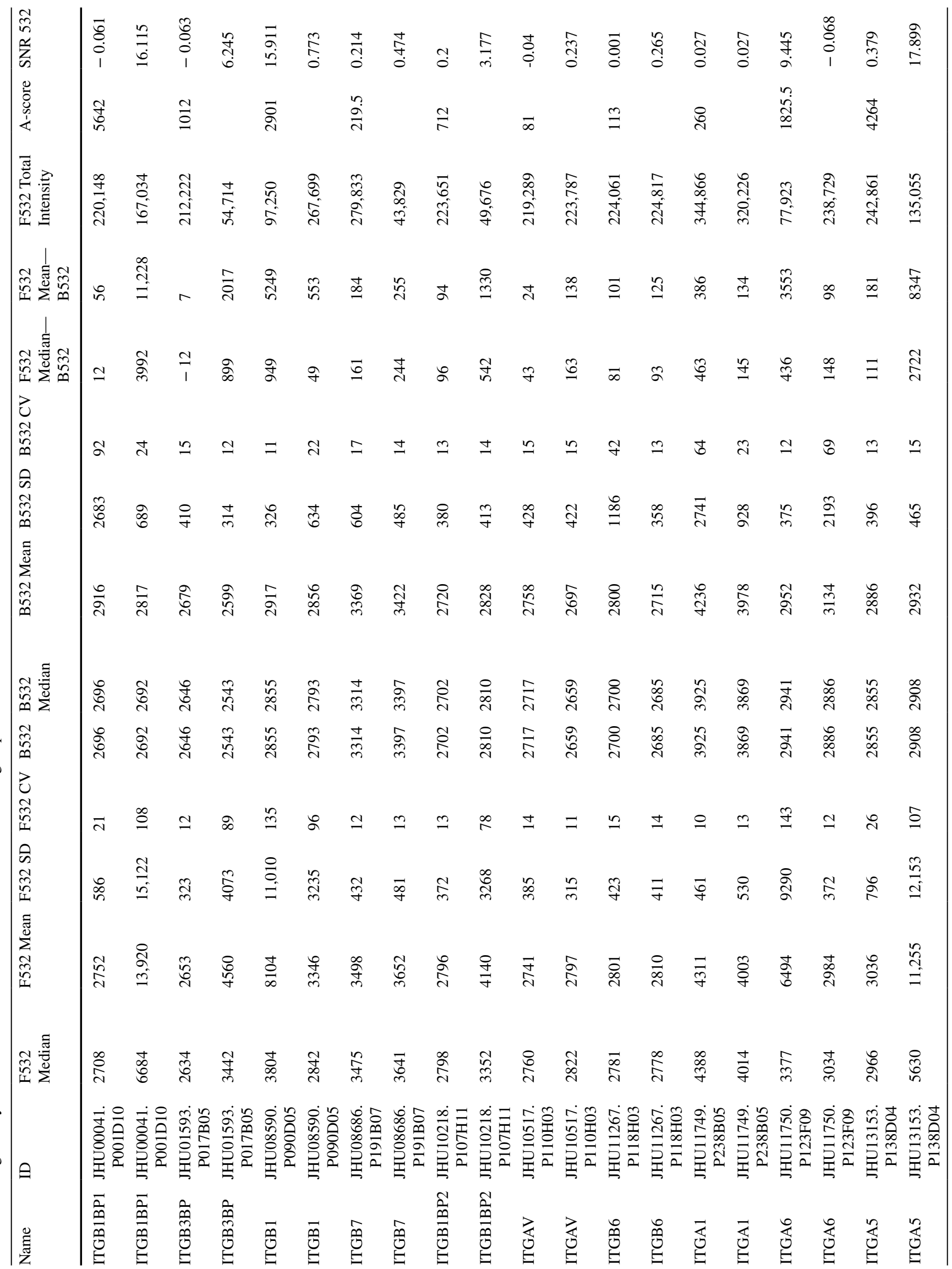




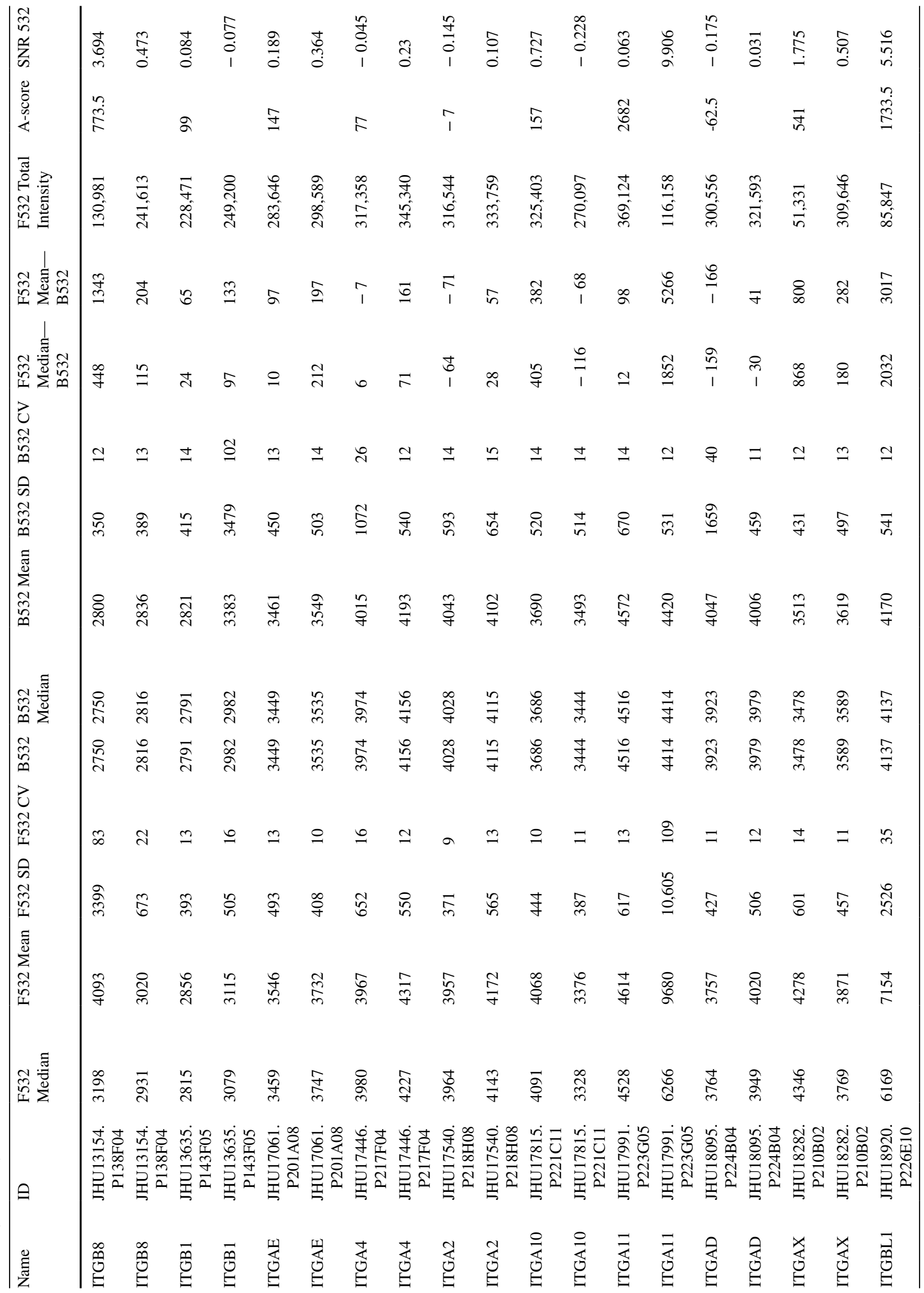




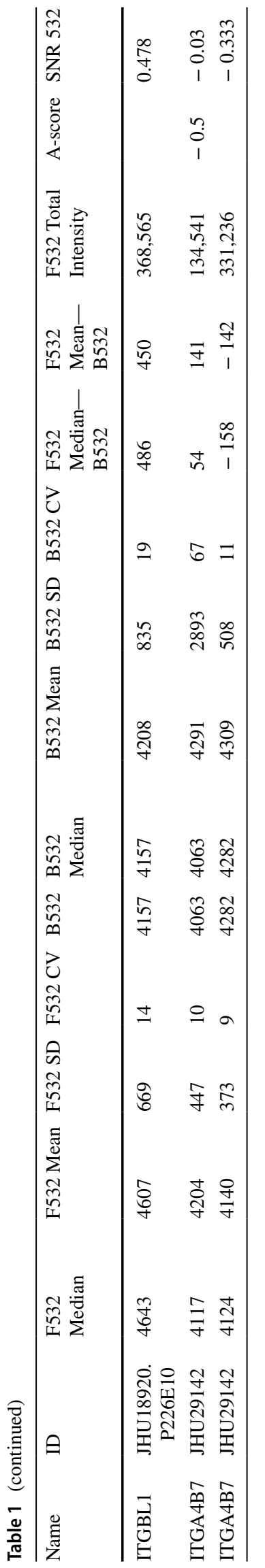

\section{Effects of caveolin, clathrin and macropinocytosis on internalization of exosomes derived from gastric epithelial cells}

We further examined whether vesicle trafficking-associated proteins including caveolin 1 and syntaxin 6 were associated with the internalization of exosomes derived from gastric epithelial cells. Vesicle trafficking-associated proteins in HFE-145, AGS, and MKN1 gastric epithelial origin cells showed expression levels similar to those in HT29 colorectal adenocarcinoma cells, H460 non-smallcell lung cancer cells, SNU449 hepatocellular carcinoma cells (Fig. 3a). However, caveolin 1 protein was not expressed in HFE-145 (Fig. 3a). AGS cells showed weak caveolin 1 expression, whereas MKN1 and non-gastric cancer cells had strong expression of caveolin 1 protein (Fig. 3a). Next, we examined the expression levels of clathrin and caveolin 1 in non-cancerous gastric mucosae and gastric cancer tissues. The expression of clathrin was slightly higher in gastric cancer tissues than non-cancerous gastric mucosae, whereas there was no significant difference in the expression of caveolin 1 proteins between non-cancerous gastric mucosae and gastric cancer tissues (Fig. 3b).

To further evaluate whether caveolin 1, clathrin, and macropinocytosis contributed to the uptake of exosomes in gastric epithelial cells, we treated AGS and MKN1 gastric cancer cells with genistein (an inhibitor of caveolin-dependent endocytosis) (27), Pitstop 2 (an inhibitor of clathrin-dependent endocytosis) [28], and 5-( $n$-ethyl$n$-isopropyl)-amiloride (EIPA), a macropinocytosis inhibitor. Notably, inhibition of clathrin and macropinocytosis markedly reduced the uptake of exosomes into AGS and MKN1 cells (Fig. 3c, d), implying that the uptake of exosomes in gastric epithelial cells might require clathrin and macropinocytosis. When we treated MKN1 cells with strong caveolin 1 expression and AGS cells with weak caveolin 1 expression with exosomes carrying GKN1 protein, exosome uptake was higher in AGS cells than that in MKN1 cells. In contrast, caveolin 1 inhibition in MKN1 cells increased the uptake of exosomes into MKN1 gastric cancer cells (Fig. 3c, d). When we further confirmed the effects of clathrin and caveolin 1 expression on the uptake of exosomes in gastric epithelial cells, knockdown of clathrin inhibited the internalization of exosomes into AGS and MKN1 cells (Fig. 3e, f). Interestingly, ectopic expression of caveolin 1 in AGS cells inhibited the internalization of exosomes, whereas knockdown of caveolin 1 in MKN1 cells increased the internalization of exosomes (Fig. 3e, f). These results indicating that caveolin 1 could inhibit the uptake of exosomes into gastric epithelial cells. 
a

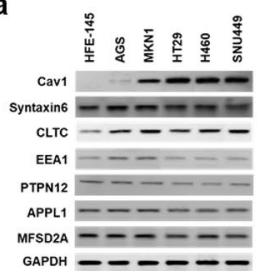

b

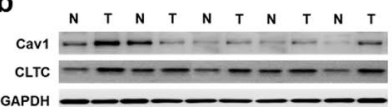

C
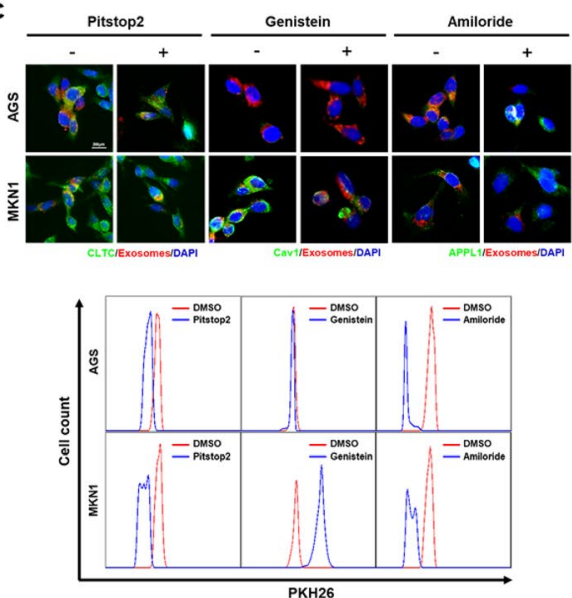

d

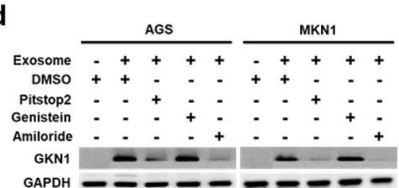

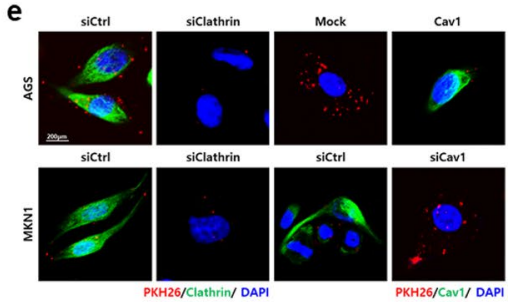
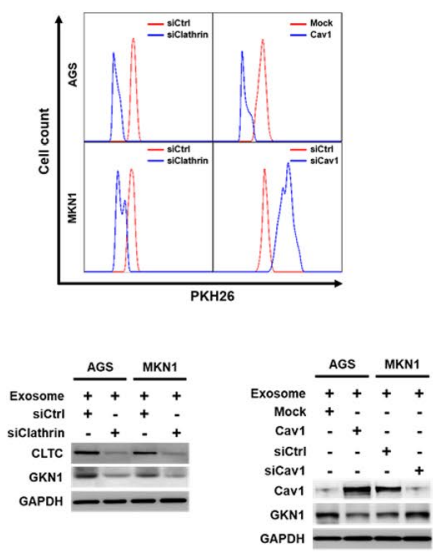

Fig. 3 Effects of caveolin, clathrin, and macropinocytosis on internalization of exosomes. a Expression levels of vesicle trafficking-associated proteins in HFE-145, AGS, MKN1, HT29, H460, and SNU449 cells. Cav1; caveolin 1, CLTC; clathrin, EEA1; early endosome antigen 1, PTPN12; protein tyrosine phosphatase non-receptor type 12, APPL1; adaptor protein, phosphotyrosine interacting with PH domain and leucine zipper 1, MFSD2A; major facilitator superfamily domain containing 2A. b The expression of clathrin and caveolin 1 proteins in corresponding non-cancerous gastric mucosae $(\mathrm{N})$ and gastric cancer (T) tissues. c Cellular uptake of exosomes following pre-treatment with $5 \mu \mathrm{M}$ pitstop $2,100 \mu \mathrm{M}$ genistein, or $50 \mu \mathrm{M}$ amiloride before addition of exosomes in AGS and MKN1 cells. d In AGS and MKN1 cells, pre-treatment with $5 \mu \mathrm{M}$ pitstop 2 and $50 \mu \mathrm{M}$ amiloride before

\section{GKN1 inhibits cell growth by downregulating Ras/ Raf/MEK/ERK pathway signaling in gastric cells}

Transwell co-culture of AGS gastric cancer cells with HFE145 gastric epithelial cells markedly inhibited viability and proliferation of AGS cancer cells (Fig. 4a), suggesting that exosomal components of HFE-145 gastric epithelial cells could inhibit cancer cells' survival and growth. Previously, we have reported that GKN1-positive exosomes derived from HFE-145 cells can significantly inhibit viability and proliferation of AGS and MKN1 gastric cancer cells [18]. To identify the molecular mechanism underlying tumor suppressor activity of GKN1, we here examined effects of GKN1 protein on the Ras/Raf/MEK/ERK kinase cascade. Expectedly, AGS cells co-cultured with HFE-145 cells showed markedly reduced expression levels of c-Myc, p-PI3K, p-Akt, HRas, b-Raf, c-Raf, and p-Erk (Fig. 4b). When AGS and MKN1 cells were treated with HFE145-derived exosomes, GKN1 decreased expression levels treatment with exosome derived from HFE-145 cells reduced expression levels of GKN1, whereas treatment with $100 \mu \mathrm{M}$ genistein increased expression levels of GKN1. e In immunofluorescent and FACS analysis, knockdown of Clathrin inhibited the internalization of exosomes into AGS and MKN1 cells. Ectopic expression of caveolin 1 in AGS cells inhibited the internalization of exosomes, whereas knockdown of caveolin 1 in MKN1 cells induced the internalization of exosomes. $\mathbf{f}$ Knockdown of clathrin in AGS and MKN1 cells treated with exosomes derived from HFE-145 cells reduced expression of GKN1 protein (left). Ectopic expression of caveolin 1 in AGS cells reduced expression level of GKN1 protein and knockdown of caveolin 1 in MKN1 cells increase expression level of GKN1 protein (right)

of c-Myc, p-PI3K, p-Akt, HRas, b-Raf, c-Raf, and p-Erk in AGS and MKN1 cells (Fig. 4c). In addition, knockdown of GKN1 with shGKN1 in HFE-145 cells increased expression of c-Myc, p-PI3K, p-Akt, HRas, b-Raf, c-Raf, and p-Erk (Fig. 4d), suggesting that GKN1 could downregulate the Ras/Raf/MEK/ERK kinase signaling pathway.

Next, we examined the binding of HRas and GKN1 proteins to determine how GKN1 regulates the Ras/Raf/MEK/ ERK kinase signaling pathway. We observed the binding of GKN1 protein to HRas in HFE-145 cells by immunoprecipitation assay (Fig. 4e). In addition, GKN1 protein inhibited the binding of HRas to b-Raf and c-Raf in AGS cells co-cultured with HFE-145 cells (Fig. 4f). Expectedly, Ras protein bound to both GTP $\gamma \mathrm{S}$ and GST-Raf1-RBD in AGS cells, but not in AGS cells co-cultured with HFE-145 cells (Fig. 4g). When we treated AGS and MKN1 cells with HFE-145-derived exosomes, GKN1 protein bound directly to HRas and completely inhibited the binding of HRas to b-Raf and c-Raf in AGS and MKN1 cells (Fig. 4h). In 
a

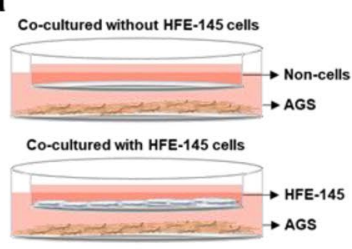

e

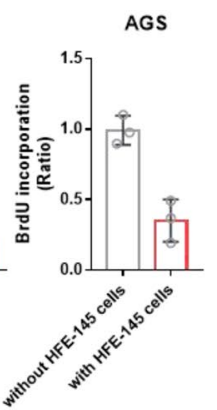

b

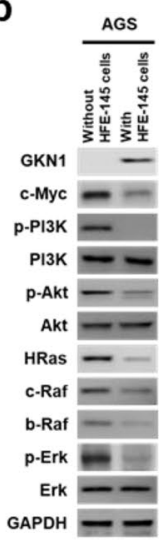

C

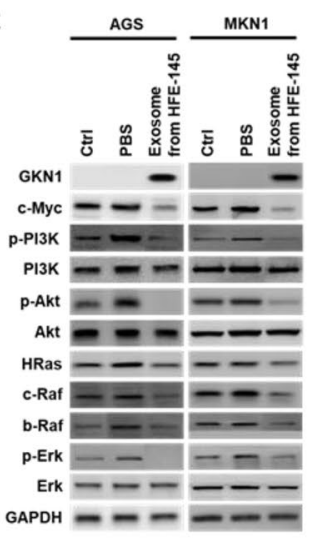

d

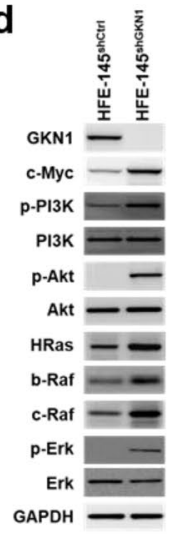

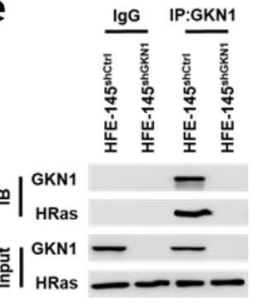

f
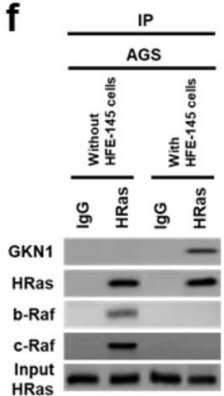

g

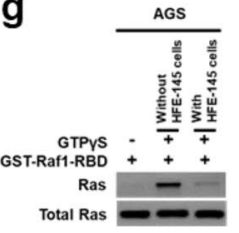

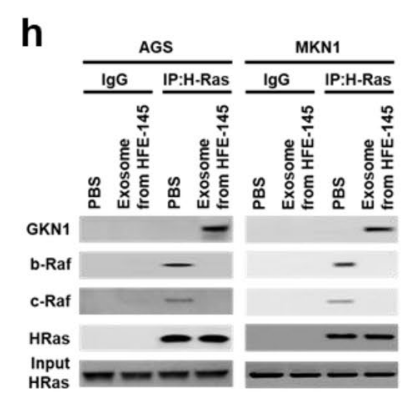

i
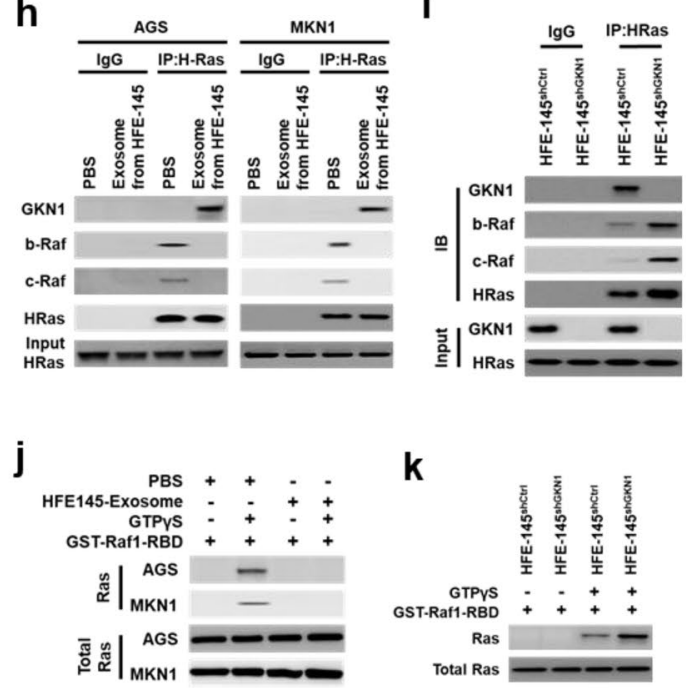

k

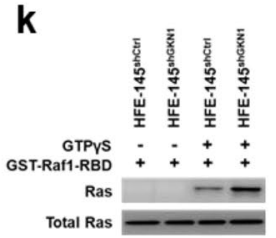

Fig. 4 GKN1 inhibits Ras/Raf/MEK/ERK signaling pathway. a Transwell co-culture system of AGS gastric cancer cells with HFE-145 immortalized normal gastric epithelial cells were used in this study. AGS and HFE-145 cells were separately cultured for $24 \mathrm{~h}$ and then co-cultured in serum-free medium for a further $96 \mathrm{~h}$ as described in Materials and methods (left panel). Transwell co-culture of AGS cells with HFE-145 cells inhibited cell viability and proliferation of AGS cells (right panel). b Expression levels of c-Myc, p-PI3K, p-Akt, HRas, b-Raf, c-Raf, and p-Erk in AGS cells co-cultured with HFE145 cells. c Treatment with exosomes derived from HFE-145 cells decreased expression levels of c-Myc, p-PI3K, p-Akt, HRas, b-Raf, c-Raf, and p-Erk in AGS and MKN1 cells. $\mathbf{d}$ Knockdown of GKN1 in HFE-145 cells increased expression levels of c-Myc, p-PI3K, p-Akt, HRas, b-Raf, c-Raf, and p-Erk. e Immunoprecipitation assay showed that GKN1 bound to HRas in HFE-145 cells. f In AGS cells co-cultured with HFE-145 cells, GKN1 bound to HRas and inhibited the binding of HRas to b-Raf and c-Raf. g Detection of active Ras (RasGTP) by pulldown assay in co-cultured AGS cells without or with HFE-145 cells. Ras activation was detected in co-cultured AGS cells without HFE-145 cells, but not detected in co-cultured AGS cells with HFE-145 cells. h Exosomes derived from HFE-145 cells inhibited the binding of HRas to b-Raf and c-Raf in AGS and MKN1 cells. i Binding of HRas to b-Raf and c-Raf was observed in GKN1 knockdown HFE-145 cells. $\mathbf{j}$ In AGS and MKN1 cells treated with PBS, Ras protein bound to GTP $\gamma \mathrm{S}$ and GST-Raf1-RBD, but exosomes derived from HFE-145 cells inhibited the binding of Ras protein to GTP $\gamma \mathrm{S}$ and GST-Raf1-RBD. $\mathbf{k}$ Binding of Ras protein to GTP $\gamma \mathrm{S}$ and GST-Raf1-RBD was found in GKN1 knockdown HFE-145 cells addition, binding of HRas to b-Raf and c-Raf was found in GKN1 knockdown HFE-145 cells (Fig. 4i). Consistently, Ras protein bound to both GTP $\gamma \mathrm{S}$ and GST-Raf1-RBD in PBS-treated AGS cells and GKN1 knockdown HFE-145 cells, whereas Ras protein did not bound to both GTP $\gamma \mathrm{S}$ and GST-Raf1-RBD in HFE-145 cells, and AGS and MKN1 cells treated with exosomes derived from HFE-145 cells (Fig. 4j, k). These findings demonstrate that normal exosomes containing GKN1 protein can inhibit the Ras activity.

When we examined the binding activity of GKN1 domains to HRas (Fig. 5a), amino terminal hydrophobic region (GKN1 $1^{\Delta 68-199}$ ) of GKN1 weakly bound to HRas, whereas the BRICHOS domain (GKN1 $1^{\Delta 1-67,165-199}$, GKN $1^{\Delta 1-67}$ ) strongly bound to HRas (Fig. 5b). In addition, Ras protein bound to both GTP $\gamma \mathrm{S}$ and GST-Raf1-RBD in AGS cells transfected with amino terminal hydrophobic region $\left(\mathrm{GKN} 1^{\triangle 68-199}\right)$ or carboxyl terminal region $\left(G K N 1^{\Delta 1-164}\right)$ of GKN1. However, Ras protein did not bind to both GTP $\gamma$ S and GST-Raf1-RBD in AGS cells transfected with the BRICHOS domain (GKN1 ${ }^{\Delta 1-67,165-199}$, GKN1 $1^{\Delta 1-67}$ ) of GKN1 (Fig. 5c). These findings demonstrate that the BRICHOS domain of GKN1 may be the 

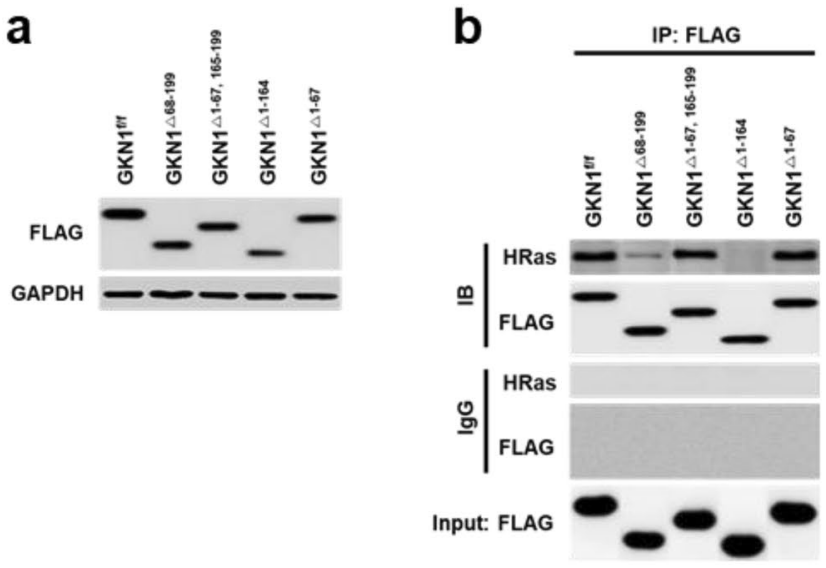

C

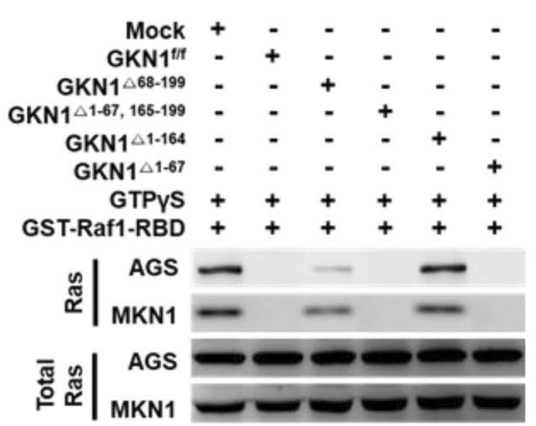

Fig. 5 Binding domain of GKN1 protein to Ras and inhibition of Ras/Raf/MEK/ERK signaling pathway by exosomes derived from HFE-145 cells. a Expression of GKN1 $1^{\mathrm{f} / \mathrm{f}}$ (full length), GKN1 ${ }^{\Delta 68-199}$, GKN1 $1^{\Delta 1-67,165-199}$, GKN1 $^{\Delta 1-164}$, and GKN1 $1^{\Delta 1-67}$ and GAPDH were determined by Western blot analysis. b Amino terminal hydrophobic region $\left(\mathrm{GKN} 1^{\Delta 68-199}\right)$ of GKN1 weakly bound to HRas, whereas the BRICHOS domain $\left(\mathrm{GKN1} 1^{\Delta 1-67,165-199}\right.$, GKN1 $\left.{ }^{\Delta 1-67}\right)$ strongly bound to HRas. $\mathbf{c}$ In AGS and MKN1 cells transfected with amino terminal hydrophobic region $\left(\mathrm{GKN} 1^{\Delta 68-199}\right)$ and carboxy terminal region $\left(\mathrm{GKN} 1^{\Delta 1-164}\right)$ of $\mathrm{GKN} 1$, Ras protein bound to GTP $\gamma \mathrm{S}$ and

physiological inhibitor of GTP binding to Ras protein in gastric epithelial cells. In addition, GKN1 decreased expression levels of c-Myc, HRas, c-Raf, b-Raf, and p-Erk in MKN1-derived xenograft tumors treated with exosomes derived from HFE-145 cells (Fig. 5d). Also, Ras protein bound to GTP $\gamma$ S and GST-Raf1-RBD in MKN1-derived xenograft tumors treated with PBS. However, exosomes derived from HFE-145 cells inhibited Ras activity (Fig. 5e), suggesting that GKN1 may induce Ras inactivation. Taken together, these results indicate that GKN1 protein in exosomes derived from gastric epithelial cells can inhibit cell growth by downregulating Ras/Raf/MEK/ ERK kinase signaling pathway.

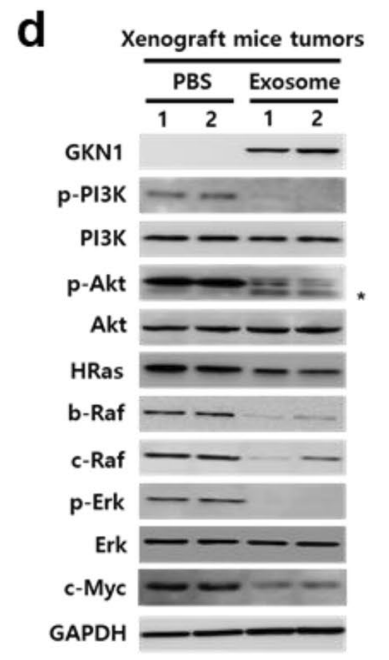

e

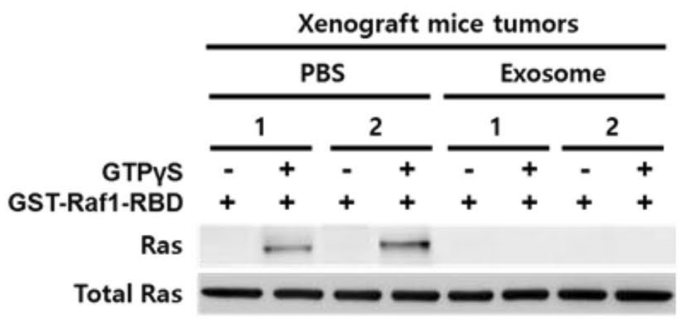

GST-Raf1-RBD, but the BRICHOS domain $\left(\mathrm{GKN} 1^{\Delta 1-67,165-199}\right.$, $G K N 1^{\Delta 1-67}$ ) of GKN1 inhibited the binding of Ras protein to GTP $\gamma \mathrm{S}$ and GST-Raf1-RBD. d Treatment with exosomes derived from HFE-145 cells decreased expression levels of c-Myc, p-PI3K, p-Akt, HRas, b-Raf, c-Raf, and p-Erk in MKN1-derived xenograft tumor tissues. e MKN1-derived xenograft tumor tissues treated with PBS, Ras protein bound to GTP $\gamma \mathrm{S}$ and GST-Raf1-RBD, but treatment with exosomes derived from HFE-145 cells inhibited the binding of Ras protein to GTP $\gamma$ S and GST-Raf1-RBD

\section{Exosomes derived from gastric epithelial cells inhibit epithelial-mesenchymal transition of gastric cancer cells}

After co-culture of AGS gastric cancer cells with HFE-145 immortalized gastric epithelial cells, we analyzed effects of GKN1-positive exosomes on EMT of AGS cells. In transwell microchemotaxis and Matrigel assays, AGS cells co-cultured with HFE-145 cells showed decreased cell migration and invasion activity (Fig. 6a, b). In Western blot analysis, AGS cells co-cultured with HFE-145 cells showed increased E-cadherin expression but reduced expression of EMT-related proteins, including N-cadherin, ZEB1, Snail, Slug and Rho-GTP (Fig. 6c). Next, we treated AGS and 

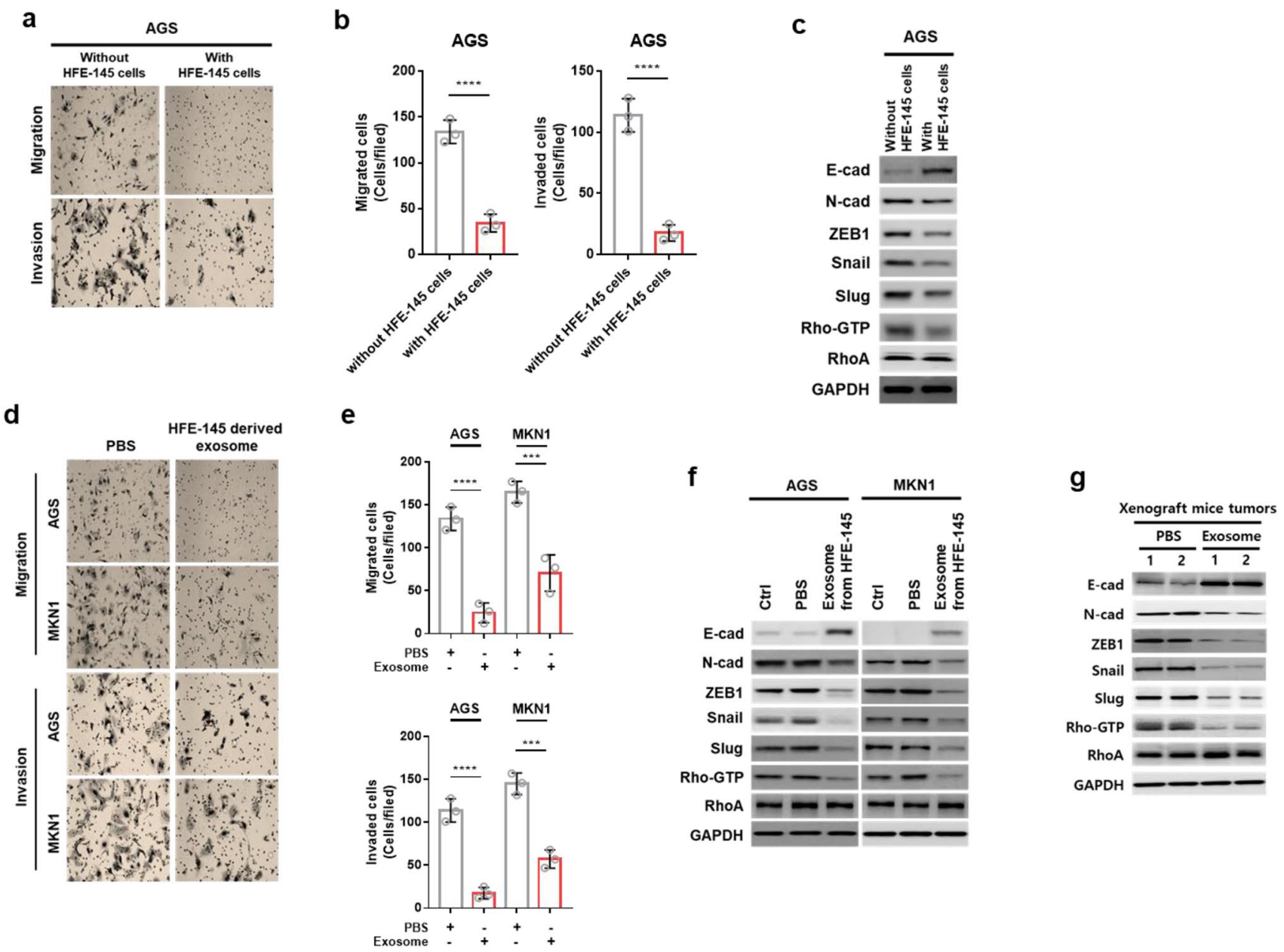

Fig. 6 Exosomes derived from gastric epithelial cells inhibit epithelial-mesenchymal transition. a, b In transwell microchemotaxis and Matrigel assays, AGS cells co-cultured with HFE-145 cells showed decreased cell migration and invasion activity, compared to AGS cells cultured without HFE-145 cells. c Expression levels of E-cadherin, N-cadherin, ZEB1, Snail, Slug, and Rho-GTP in AGS cells cocultured without or with HFE-145 cells. d, e Migration and invasion

MKN1 cells with exosomes derived from HFE-145 cells to investigate whether normal exosomes inhibited the progression of gastric cancer cells. As shown in Fig. 6d, e, migration and invasion of gastric cancer cells were dramatically reduced in AGS and MKN1 cells treated exosomes derived from HFE-145 cells, compared to those of control cells. Similar to co-culture of AGS with HFE-145 cells, treatment with exosomes derived from HFE-145 cells also showed increased E-cadherin expression but reduced expression of N-cadherin, ZEB1, Snail, Slug, and Rho-GTP in AGS, MKN1 cells, and xenograft tumors tissues (Fig. 6f, g). These findings suggest that GKN1 protein in exosomes derived from gastric epithelial cells can inhibit gastric cancer cell migration and invasion by regulating EMT-related protein expression. activity of AGS and MKN1 cells were reduced in HFE-145-derived exosome-treated cells, compared to that of PBS-treated cells. f, $\mathbf{g}$ Expression levels of E-cadherin, N-cadherin, ZEB1, Snail, Slug, and Rho-GTP in AGS and MKN1 cells (f), and MKN1-derived xenograft tumor tissues (g) treated with PBS or exosomes derived from HFE145 cells

\section{Discussion}

Here, we found that the uptake of exosomes derived from HFE-145 immortalized gastric epithelial cells occurred only in HFE-145, AGS, and MKN1 cells, but not in colon, liver, or lung cancer cells. In addition, expression levels of integrin $\alpha 5, \alpha \mathrm{X}, \alpha 6$, and $\alpha \varepsilon$ were higher in gastric cells and exosomes derived from HFE-145, AGS, and MKN1 cells than those in exosomes derived from colon, liver, and lung cancer cells. In particular, integrin $\alpha 6$ and $\alpha \mathrm{X}$ were expressed only in HFE-145, AGS, and MKN1 cells, and stomach tissues. Knockdown of integrin $\alpha 6$ and $\alpha \mathrm{X}$ with silntegrin $\alpha 6$ and silntegrin $\alpha X$ in AGS and MKN1 cells dramatically inhibited the internalization of PKH26-positive exosomes into AGS and MKN1 cells' cytoplasms, respectively. Furthermore, 
ectopic expression of both integrin $\alpha 6$ and $\alpha \mathrm{X}$ in HT29 colon cancer cells, H460 lung cancer cells, and SNU449 hepatocellular carcinoma cells markedly induced internalization of exosomes derived from HFE-145 gastric epithelial cells. Also, exosomes derived from HT29 cells, H460 cells, and SNU449 cells ectopically expressing integrin $\alpha 6$ and $\alpha \mathrm{X}$ were internalized into AGS cells. These results suggest that exosome uptake depends on the expression pattern of integrin proteins of recipient cells and exosomes, and that gastric-specific uptake of exosomes derived from gastric epithelial cells requires higher expression of $\alpha 6$ and $\alpha \mathrm{X}$ integrin proteins in both gastric epithelial cells and exosomes.

Next, we further investigated whether macropinocytosis, clathrin-mediated endocytosis, and caveolin-mediated endocytosis modulated the internalization of exosomes derived from gastric epithelial cells. Notably, no significant difference was found in the expression of vesicle traffickingassociated proteins, including syntaxin 6 or clathrin heavy chain 1 (CLTC), between HFE-145 gastric epithelial cells and cancer cells originating from stomach, lung, liver, and colon. Of these, caveolin 1 protein was not expressed in HFE-145 while AGS cells showed weak cavolin 1 expression. However, MKN1 and other cancer cells demonstrated strong expression of caveolin 1 protein. In human stomach tissues, expression of clathrin was slightly higher in gastric cancers than corresponding non-cancerous gastric mucosae, whereas there was no significant difference in the expression of caveolin 1 proteins between non-cancerous gastric mucosae and gastric cancer tissues. Interestingly, inhibition of clathrin and macropinocytosis markedly reduced the uptake of exosomes into AGS and MKN1 cells. Caveolin inhibition with genistein and siCaveolin 1 increased the uptake of exosomes carrying GKN1 protein into MKN1 gastric cancer cells, whereas ectopic expression of caveolin 1 in AGS gastric cancer cells reduced the uptake of exosomes. These results are consistent with previous data showing that efficient exosome uptake is mediated by clathrin-dependent endocytosis [29] and that caveolin 1 negatively regulates endocytosis of exosomes [16]. Thus, we can conclude that the uptake of exosomes in gastric epithelial cells may require clathrin and macropinocytosis and that caveolin 1 can inhibit the uptake of exosome into gastric epithelial cells.

Cellular interactions with exosomes induce cell proliferation, apoptosis, invasion, and metastasis. Previously, we have reported that exosome-associated GKN1 protein can significantly inhibit viability and proliferation of AGS and MKN1 gastric cancer cells and reduce tumor volume and tumor weight of nude mice-bearing MKN1 xenograft tumors [18]. Here, co-localization of GKN1 protein with exosomes derived from HFE-145 cells was found in cytoplasms of AGS and MKN1 cells treated with PKH26 labeled exosomes. To further study the effect of exosomesassociated GKN1 protein on maintenance of homeostasis of gastric epithelium, we next sought to define the molecular pathway associated with cellular proliferation using a transwell co-culture system. Previously, we have reported that GKN1 can downregulate H. pylori CagA-induced overexpression of Ras and Raf family proteins in gastric cells and human gastric mucosae [30]. It is well known that the $\mathrm{Ras} / \mathrm{Raf} / \mathrm{MEK} / \mathrm{ERK}$ kinase cascade is an essential effector cascade required for Ras GTPase signaling [31]. To trigger the Ras/Raf/MEK/ERK signaling pathway, growth factor needs to bind to its cognate receptor which in turn activates Ras-GTP by binding of GRB2 and SOS to the growth factor receptor [32]. Raf, a serine/threonine protein kinase and Ras effector, can promote protein phosphorylation via MEK/ ERK activation [33]. Its upstream kinases MEK1/2 can activate ERK1/2 kinases known to mediate cell proliferation and apoptosis [34]. ERK1/2 can induce phosphorylation of various transcription factors such as Ets-1, c-Jun, c-Myc, and NF-kB [35]. p-ERK is a key downstream target of the Ras/Raf/MEK/ERK signaling pathway [33]. This cascade is involved in cell biology including cell proliferation, apoptosis, differentiation, and migration. Abnormal activation of this pathway is commonly detected in human cancers. It also influences chemotherapeutic drug resistance [32]. In the present study, we uncovered a new role of exosomes-associated GKN1 protein for inactivation of the Ras/Raf/MEK/ERK signaling pathway. In the transwell system, co-culture of AGS gastric cancer cells with HFE-145 immortalized gastric epithelial cells markedly inhibited viability and proliferation of AGS cancer cells and reduced expression levels of c-Myc, p-PI3K, p-Akt, HRas, b-Raf, c-Raf, and p-Erk. In addition, GKN1, especially the BRICHOS domain, bound to HRas, inhibited binding of HRas to b-Raf and c-Raf, and finally decreased the expression of p-ERK in AGS cells, MKN1 cells, and xenograft tumors treated with exosomes derived from HFE-145 cells, indicating that GKN1 protein could suppress cell proliferation of gastric epithelial cells by inhibiting the Ras/Raf/MEK/ERK signaling pathway. The BRICHOS superfamily consists of four distinct regions: hydrophobic, linker, BRICHOS, and C-terminal [36]. GKN proteins contain the BRICHOS domain, a $\mathrm{COOH}$-terminal segment and hydrophobic $\mathrm{NH}_{2}$-terminal signal peptide [37]. In the previous study, we found that BRICHOS domain is the main domain for the GKN1 tumor suppressor function [25]. Taken together, these findings strongly suggest that secreted exosomal GKN1 protein from gastric epithelial cells might be internalized into gastric cells, thus altering a set of signaling pathways involved in the regulation of cell growth.

Interestingly, GKN1 protein inhibited gastric cancer cell migration and invasion by downregulating c-Myc, RhoA, Snail, and Slug expression and inactivating NF-kB pathway [38]. Consistently, co-culture of AGS gastric cancer cells with HFE-145 immortalized gastric epithelial cells showed 
dramatically decreased migration and invasion of AGS cells. In addition, increased E-cadherin expression and reduced expression of EMT-related proteins including N-cadherin, ZEB1, Snail, Slug, and Rho-GTP were detected in both AGS gastric cancer cells co-cultured with HFE-145 cells and xenograft tumors treated with exosomes derived from HFE-145 cells. These data indicate that exosome-associated GKN1 protein can suppress migration and invasion of gastric cancer cells by inhibiting EMT.

In summary, gastric-specific uptake of stomach-derived exosomes requires integrin $\alpha 6$ and $\alpha \mathrm{X}$ proteins in gastric cells and exosomes derived from gastric cells. Clathrin and macropinocytosis can increase the uptake of exosomes into gastric epithelial cells, but caveolin can inhibit the uptake of exosomes. In the transwell co-culture, exosomes derived from HFE-145 cells markedly inhibited viability and proliferation of AGS and MKN1 cancer cells. GKN1 protein bound to HRas and downregulated PI3K/Akt and HRas/ Raf/MEK/ERK signaling pathways. In addition, exosomeassociated GKN1 protein suppressed migration and invasion of gastric cancer cells by inhibiting EMT. Thus, we can conclude that gastric-specific uptake of exosomes derived from gastric epithelial cells requires integrin $\alpha 6$ and $\alpha \mathrm{X}$ proteins in both gastric epithelial cells and exosomes and that exosome-associated GKN1 protein can inhibit gastric carcinogenesis by downregulating the HRas/Raf/MEK/ERK signaling pathway.

Acknowledgements This study was supported by the Basic Science Research Program through the National Research Foundation of Korea (NRF) funded by the Ministry of Education, Science and Technology (2018R1A2A2A14019713). We thank Dr. Seong Yeob Ryu, Department of Gastroenterologic Surgery, Chonnam National University Hwasun Hospital, 160, Ilsim-ri, Hwasun-eup, Hwasun-gun, Jeollanamdo, 519-809, Korea, for providing the human samples with clinical information.

Author contributions JHY: concept and design; provision of study materials; data collection, analysis, and interpretation; article writing; and final approval of the article. WSP: concept and design; provision of study materials; article writing; and final approval of the article. HA and DTS: provided the HFE-145 cells before publication; and final approval of the article. SWN: manuscript drafting for important intellectual content; and final approval of the article. $\mathrm{HH}$ : performed in vivo experiments; and final approval of the article.

\section{Compliance with ethical standards}

Conflict of interest The authors have declared no conflicts of interest. Dr. Park reports grants from The Basic Science Research Program through the National Research Foundation of Korea (NRF) funded by the Ministry of Education, Science and Technology (2018R1A2A2A14019713 to W.S.P).

Ethical standards

All procedures followed were in accordance with the ethical standards of the responsible committee on human experimentation (institutional and national) and with the Helsinki Declaration of 1964 and later versions. Informed consent to be included in the study, or the equivalent, was obtained from all patients. For studies with animals, all institutional and national guidelines for the care and use of laboratory animals were followed.

Ethical standards All procedures followed were in accordance with the ethical standards of the responsible committee on human experimentation (institutional and national) and with the Helsinki Declaration of 1964 and later versions. Informed consent to be included in the study, or the equivalent, was obtained from all patients. For studies with animals, all institutional and national guidelines for the care and use of laboratory animals were followed.

\section{References}

1. Simons M, Raposo G. Exosomes-vesicular carriers for intercellular communication. Curr Opin Cell Biol. 2009;21(4):575-81. https://doi.org/10.1016/j.ceb.2009.03.007 (Epub 2009/05/16).

2. Mathivanan S, Ji H, Simpson RJ. Exosomes: extracellular organelles important in intercellular communication. J Proteomics. 2010;73(10):1907-20. https://doi.org/10.1016/j.jprot.2010.06.006 (Epub 2010/07/06).

3. Hessvik NP, Llorente A. Current knowledge on exosome biogenesis and release. Cell Mol Life Sci. 2018;75(2):193-208. https:// doi.org/10.1007/s00018-017-2595-9 (Epub 2017/07/25).

4. Ruivo CF, Adem B, Silva M, Melo SA. The biology of cancer exosomes: insights and new perspectives. Cancer Res. 2017;77(23):6480-8. https://doi.org/10.1158/0008-5472.CAN17-0994 (Epub 2017/11/23).

5. Brinton LT, Sloane HS, Kester M, Kelly KA. Formation and role of exosomes in cancer. Cell Mol Life Sci. 2015;72(4):659-71. https://doi.org/10.1007/s00018-014-1764-3 (Epub 2014/10/23).

6. Seo N, Shirakura Y, Tahara Y, Momose F, Harada N, Ikeda H, et al. Activated CD8(+) T cell extracellular vesicles prevent tumour progression by targeting of lesional mesenchymal cells. Nat Commun. 2018;9(1):435. https://doi.org/10.1038/s4146 7-018-02865-1 (Epub 2018/02/01)

7. Escrevente C, Keller S, Altevogt P, Costa J. Interaction and uptake of exosomes by ovarian cancer cells. BMC Cancer. 2011;11:108. https://doi.org/10.1186/1471-2407-11-108 (Epub 2011/03/29).

8. Hoshino A, Costa-Silva B, Shen TL, Rodrigues G, Hashimoto A, Tesic Mark M, et al. Tumour exosome integrins determine organotropic metastasis. Nature. 2015;527(7578):329-35. https ://doi.org/10.1038/nature15756 (Epub 2015/11/03).

9. McKelvey KJ, Powell KL, Ashton AW, Morris JM, McCracken SA. Exosomes: mechanisms of uptake. J Circ Biomark. 2015;4:7. https://doi.org/10.5772/61186 (Epub 2015/07/17).

10. Berditchevski F, Zutter MM, Hemler ME. Characterization of novel complexes on the cell surface between integrins and proteins with 4 transmembrane domains (TM4 proteins). Mol Biol Cell. 1996;7(2):193-207. https://doi.org/10.1091/mbc.7.2.193 (Epub 1996/02/01).

11. Feng D, Zhao WL, Ye YY, Bai XC, Liu RQ, Chang LF, et al. Cellular internalization of exosomes occurs through phagocytosis. Traffic. 2010;11(5):675-87 (Epub 2010/02/09).

12. Nakase I, Kobayashi NB, Takatani-Nakase T, Yoshida T. Active macropinocytosis induction by stimulation of epidermal growth factor receptor and oncogenic Ras expression potentiates cellular uptake efficacy of exosomes. Sci Rep. 2015;5:10300. https://doi. org/10.1038/srep10300 (Epub 2015/06/04).

13. Tian T, Zhu YL, Zhou YY, Liang GF, Wang YY, Hu FH, et al. Exosome uptake through clathrin-mediated endocytosis and macropinocytosis and mediating miR-21 delivery. J Biol Chem. 
2014;289(32):22258-67. https://doi.org/10.1074/jbc.M114.58804 6 (Epub 2014/06/22).

14. Nanbo A, Kawanishi E, Yoshida R, Yoshiyama H. Exosomes derived from Epstein-Barr virus-infected cells are internalized via caveola-dependent endocytosis and promote phenotypic modulation in target cells. J Virol. 2013;87(18):10334-47. https://doi. org/10.1128/JVI.01310-13 (Epub 2013/07/19).

15. Hazan-Halevy I, Rosenblum D, Weinstein S, Bairey O, Raanani P, Peer D. Cell-specific uptake of mantle cell lymphoma-derived exosomes by malignant and non-malignant B-lymphocytes. Cancer Lett. 2015;364(1):59-69. https://doi.org/10.1016/j.canle t.2015.04.026 (Epub 2015/05/03).

16. Svensson KJ, Christianson HC, Wittrup A, Bourseau-Guilmain E, Lindqvist E, Svensson LM, et al. Exosome uptake depends on ERK1/2-heat shock protein 27 signaling and lipid Raft-mediated endocytosis negatively regulated by caveolin-1. J Biol Chem. 2013;288(24):17713-24. https://doi.org/10.1074/jbc.M112.44540 3 (Epub 2013/05/09).

17. Martin TE, Powell CT, Wang Z, Bhattacharyya S, Walsh-Reitz $\mathrm{MM}$, Agarwal K, et al. A novel mitogenic protein that is highly expressed in cells of the gastric antrum mucosa. Am J Physiol Gastrointest Liver Physiol. 2003;285(2):G332-G34343. https:// doi.org/10.1152/ajpgi.00453.2002 (Epub 2003/07/10).

18. Yoon JH, Ham IH, Kim O, Ashktorab H, Smoot DT, Nam SW, et al. Gastrokine 1 protein is a potential theragnostic target for gastric cancer. Gastric Cancer. 2018;21(6):956-67. https://doi. org/10.1007/s 10120-018-0828-8 (Epub 2018/04/29).

19. Yoshikawa Y, Mukai H, Hino F, Asada K, Kato I. Isolation of two novel genes, down-regulated in gastric cancer. Jpn J Cancer Res. 2000;91(5):459-63. https://doi.org/10.1111/j.1349-7006.2000. tb00967.x (Epub 2000/06/03).

20. Xing R, Cui JT, Xia N, Lu YY. GKN1 inhibits cell invasion in gastric cancer by inactivating the NF-kappaB pathway. Discov Med. 2015;19(103):65-71 (Epub 2015/03/01).

21. Yoon JH, Choi WS, Kim O, Park WS. The role of gastrokine 1 in gastric cancer. J Gastric Cancer. 2014;14(3):147-55. doi: 10.5230/ jgc.2014.14.3.147. (Epub 2014/10/21)

22. Yoon JH, Kang YH, Choi YJ, Park IS, Nam SW, Lee JY, et al. Gastrokine 1 functions as a tumor suppressor by inhibition of epithelial-mesenchymal transition in gastric cancers. J Cancer Res Clin Oncol. 2011;137(11):1697-704. https://doi.org/10.1007/ s00432-011-1051-8 (Epub 2011/09/08).

23. Yoon JH, Song JH, Zhang C, Jin M, Kang YH, Nam SW, et al. Inactivation of the Gastrokine 1 gene in gastric adenomas and carcinomas. J Pathol. 2011;223(5):618-25. https://doi.org/10.1002/ path.2838 (Epub 2011/02/23).

24. Yoon JH, Choi YJ, Choi WS, Ashktorab H, Smoot DT, Nam SW, et al. GKN1-miR-185-DNMT1 axis suppresses gastric carcinogenesis through regulation of epigenetic alteration and cell cycle. Clin Cancer Res. 2013;19(17):4599-610. https://doi. org/10.1158/1078-0432.CCR-12-3675 Epub 2013/07/13.

25. Yoon JH, Choi YJ, Choi WS, Nam SW, Lee JY, Park WS. Functional analysis of the NH2-terminal hydrophobic region and BRICHOS domain of GKN1. Biochem Biophys Res Commun. 2013;440(4):689-95. https://doi.org/10.1016/j.bbrc.2013.09.123 (Epub 2013/10/09).

26. Yoon JH, Choi WS, Kim O, Choi SS, Lee EK, Nam SW, et al. NKX63 controls gastric differentiation and tumorigenesis. Oncotarget. 2015;6(29):28425-39. https://doi.org/10.18632/oncotarget .4952Epub 2015/09/01.
27. Puri V, Watanabe R, Singh RD, Dominguez M, Brown JC, Wheatley CL, et al. Clathrin-dependent and -independent internalization of plasma membrane sphingolipids initiates two Golgi targeting pathways. J Cell Biol. 2001;154(3):535-47. https://doi. org/10.1083/jcb.200102084 (Epub 2001/08/02).

28. von Kleist L, Stahlschmidt W, Bulut H, Gromova K, Puchkov $\mathrm{D}$, Robertson MJ, et al. Role of the clathrin terminal domain in regulating coated pit dynamics revealed by small molecule inhibition. Cell. 2011;146(3):471-84. https://doi.org/10.1016/j. cell.2011.06.025 (Epub 2011/08/06).

29. Horibe S, Tanahashi T, Kawauchi S, Murakami Y, Rikitake Y. Mechanism of recipient cell-dependent differences in exosome uptake. BMC Cancer. 2018;18(1):47. https://doi.org/10.1186/ s12885-017-3958-1 (Epub 2018/01/08).

30. Yoon JH, Seo HS, Choi SS, Chae HS, Choi WS, Kim O, et al. Gastrokine 1 inhibits the carcinogenic potentials of Helicobacter pylori CagA. Carcinogenesis. 2014;35(11):2619-29. https://doi. org/10.1093/carcin/bgu199 (Epub 2014/09/23).

31. Aoki Y, Niihori T, Inoue S, Matsubara Y. Recent advances in RASopathies. J Hum Genet. 2016;61(1):33-9. https://doi. org/10.1038/jhg.2015.114 (Epub 2015/10/09).

32. McCubrey JA, Steelman LS, Chappell WH, Abrams SL, Wong EW, Chang F, et al. Roles of the Raf/MEK/ERK pathway in cell growth, malignant transformation and drug resistance. Biochim Biophys Acta. 2007;1773(8):1263-84. https://doi.org/10.1016/j. bbamcr.2006.10.001 (Epub 2006/11/28).

33. Li L, Zhao GD, Shi Z, Qi LL, Zhou LY, Fu ZX. The Ras/Raf/ MEK/ERK signaling pathway and its role in the occurrence and development of HCC. Oncol Lett. 2016;12(5):3045-50. https:// doi.org/10.3892/ol.2016.5110 (Epub 2016/12/03).

34. Wei F, Yan J, Tang D. Extracellular signal-regulated kinases modulate DNA damage response-a contributing factor to using MEK inhibitors in cancer therapy. Curr Med Chem. 2011;18(35):547682 (Epub 2011/11/18).

35. Nakano H, Shindo M, Sakon S, Nishinaka S, Mihara M, Yagita $\mathrm{H}$, et al. Differential regulation of IkappaB kinase alpha and beta by two upstream kinases, NF-kappaB-inducing kinase and mitogen-activated protein kinase/ERK kinase kinase-1. Proc Natl Acad Sci USA. 1998;95(7):3537-42. https://doi.org/10.1073/ pnas.95.7.3537 (Epub 1998/05/09).

36. Sanchez-Pulido L, Devos D, Valencia A. BRICHOS: a conserved domain in proteins associated with dementia, respiratory distress and cancer. Trends Biochem Sci. 2002;27(7):329-32. https://doi. org/10.1016/s0968-0004(02)02134-5 (Epub 2002/07/13).

37. Menheniott TR, Kurklu B, Giraud AS. Gastrokines: stomach-specific proteins with putative homeostatic and tumor suppressor roles. Am J Physiol Gastrointest Liver Physiol. 2013;304(2):G109-G121121. https://doi.org/10.1152/ajpgi.00374 .2012 (Epub 2012/11/17).

38. Yoon JH, Choi WS, Kim O, Choi BJ, Nam SW, Lee JY, et al. Gastrokine 1 inhibits gastric cancer cell migration and invasion by downregulating RhoA expression. Gastric Cancer. 2017;20(2):274-85. https://doi.org/10.1007/s10120-016-0617-1 (Epub 2016/06/03).

Publisher's Note Springer Nature remains neutral with regard to jurisdictional claims in published maps and institutional affiliations. 